\title{
Comparison of Female Shape Analysis Methods for the Development of a New Sizing System
}

\author{
James M. WEBSTER, Jérémy CORNOLO, Yohann KELKEL \\ Oxylane Research, Lille, France
}

\begin{abstract}
A key factor affecting consumer satisfaction with sportswear is garment fit and in turn comfort. Currently there is a large volume of research regarding the use of $3 \mathrm{D}$ body scanning and its incorporation into sizing systems and garment development. It is a common belief that to maximise the fit of a garment, both size and form must be considered, however, some companies still base their sizing systems on a single body type/ shape. This paper aimed to categorise female body form into different shapes through the use of two evaluation techniques:

1) Determination of body shape according to pre-defined ratio rules for the width of the chest, waist and hips (silhouette shape),

2) Determination of body shape according to ratio rules based on the chest, waist and hip circumferences,

Each method was used to evaluate 300 European scans, which were collected using a TC2 scanner.

The results from both analysis methods identified that over $95 \%$ of the population could be categorised into one of 4 body types. When using analysis method 1 (silhouette shape) the 4 dominant forms were hourglass $(11 \%)$, bottom heavy hourglass $(30 \%)$, rectangle $(5 \%)$ and spoon $(49 \%)$. Whereas, through the use of method 2 the forms identified were hourglass (36\%), soft hourglass $(34 \%)$, spoon $(10 \%)$ and rectangle $(15 \%)$. To further analyse the results, a shape analysis was conducted by comparing width and depth (front to back) measurement at each location. These results identified large variations in form within shape categories when using the single width measurement. The variation in shape was significantly reduced when circumference measurements were used. Based on the results from method 2, the relationship between size and shape was examined, identifying that as size increases the chest - waist and waist - hip ratio needs to decrease. These ratio changes result in a gradual change from hourglass to rectangle shape. As a result a better fit could be potentially achieved for up to $25 \%$ of the target population.
\end{abstract}

Keywords: 3d body scanning, Female body form, shape analysis.

\section{Introduction}

A key factor affecting consumer satisfaction with sportswear is garment fit and in turn comfort [1]. The analysis of clothing fit can be traced back as far as the twelfth century where "well-cut through the body: fitted clothing" was interpreted as an image of nobility [2]. Since then clothing fit has been an area which has been highly researched as it has been identified as an extremely complex concept due to the ambiguous and multi faceted relationships between different clothing layers and the body [3]. Despite this, several clothing companies continue to develop sizing systems based on simple statistical methodologies. A common method of sizing system development is for a base size to be developed, either based on an individuals measurements or a combination of several people's measurements, that are seen to represent the average person of a population, then linear gradation of each measurement is used to develop an array of sizes [4]. The use of linear gradation makes the development of a sizing system relatively simple and in turn, quick. However, previous studies have identified that the use of these methods alone are inadequate, resulting in over $50 \%$ of women stating they can not find clothing with a good fit, with these sizing issues being more predominant the further they are from the base size $[3,5]$.

With the increase in ready to wear garments (RTW) sizing is becoming more problematic, with a large volume of research being conducted to enable more accurate and detailed sizing systems to be developed. Through the use of 3D scanning, larger studies are being conducted to enable a bigger proportion of the target population to be used when developing sizing systems [6]. Although, increasing the survey size, measurement accuracy and repeatability are beneficial, with regards to the suitability of sizing systems, the underlying issue of how to analyse the data and best represent the population within one sizing system remains. 
One of the key factors affecting clothing fit is body shape, with variation between individuals and between sizing classifications having a large affect on perceived fit and in turn comfort and satisfaction. Several studies have analysed female form with regards to body proportions of the silhouette, from the front view. Within these studies female form has been categorised into either a set of geometric shapes such as hourglass, oval and rectangle as shown in figure 1 , letter figures $A, X, V, H, O$, or fruits for example pear, or apple.

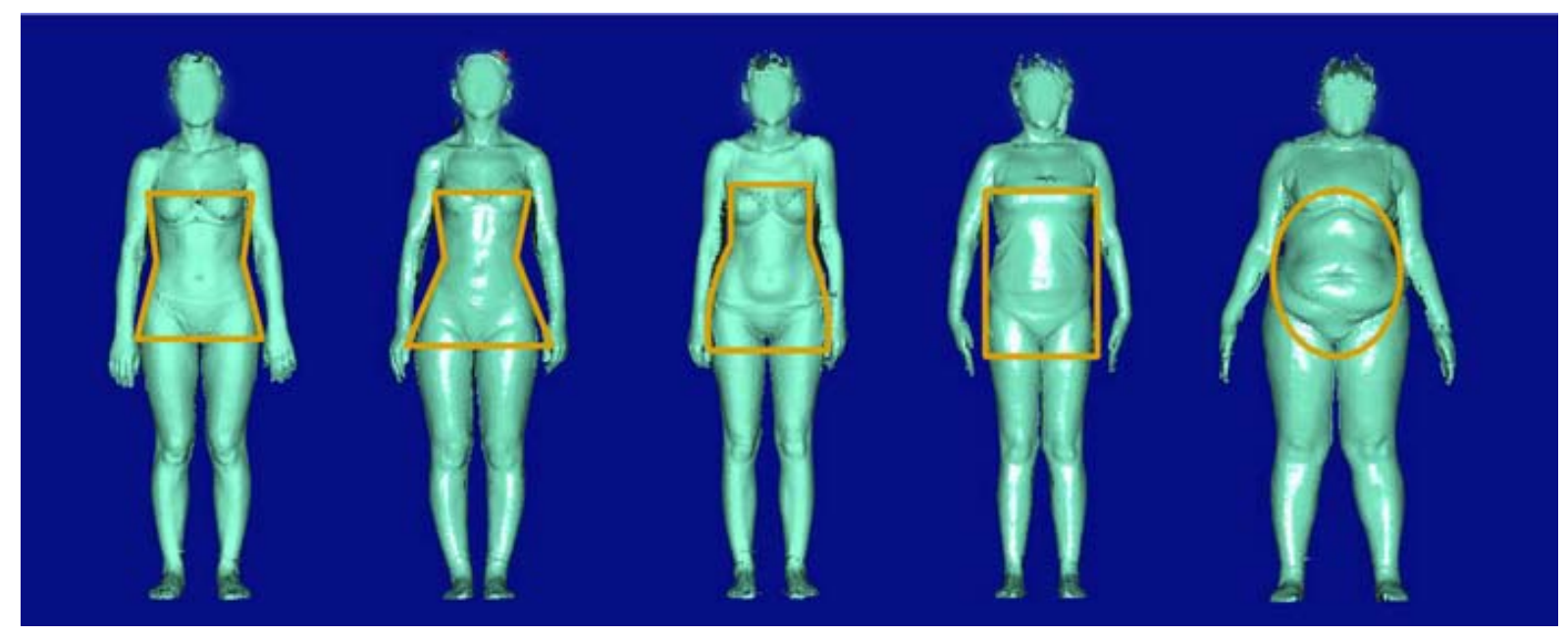

Fig. 1. Example of female form represented by silhouette shape [7].

The categorisation of female forms and the division of target populations into these shapes can enable a greater understanding of shape variation within and between sizes, with these differences being reflected in the sizing system, and by moving away from the basic linear gradation, a greater fit can be acquired. However, simply using the silhouette shape rather than measurements that are believed to provide a better understanding of the $3 \mathrm{D}$ form is questionable, therefore, this paper aimed to determine the best method to categorise female body form. To do this the following methods of categorisation were analysed:

1) Categorisation of body shape according to pre-defined ratio rules for the width of the chest, waist and hips (silhouette shape),

2) Categorisation of body shape according to ratio rules based on the chest, waist and hip circumferences,

and from this analysis identify which technique provides a greater understanding of female form variation and the relationship between size and shape.

\section{Methodology}

\subsection{Data collection}

A scan campaign was conducted in Madrid using a TC2 NX16 scanner, a white light scanner with a point accuracy of less than $1 \mathrm{~mm}$. 315 full body scans were collected of women aged 18-65, with a mean age of $35.2 \pm 11.2$ years, mean weight of $64.4 \pm 12.3 \mathrm{~kg}$ and a mean height of $1.63 \pm 0,08 \mathrm{~m}$.

\subsection{Measurement extraction}

Prior to data extraction each scan was visually checked, with all scans which were incomplete or had obvious errors discarded (e.g. figure 2). This resulted in 15 scans being removed from the database. 


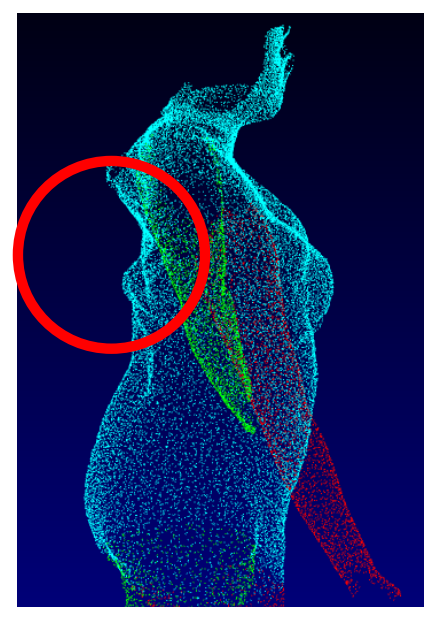

Fig. 2: Example of a discarded scan.

For each scan 9 measurements were extracted, these were circumference, width and depth at the following locations, chest, waist and hip (Figure 3).

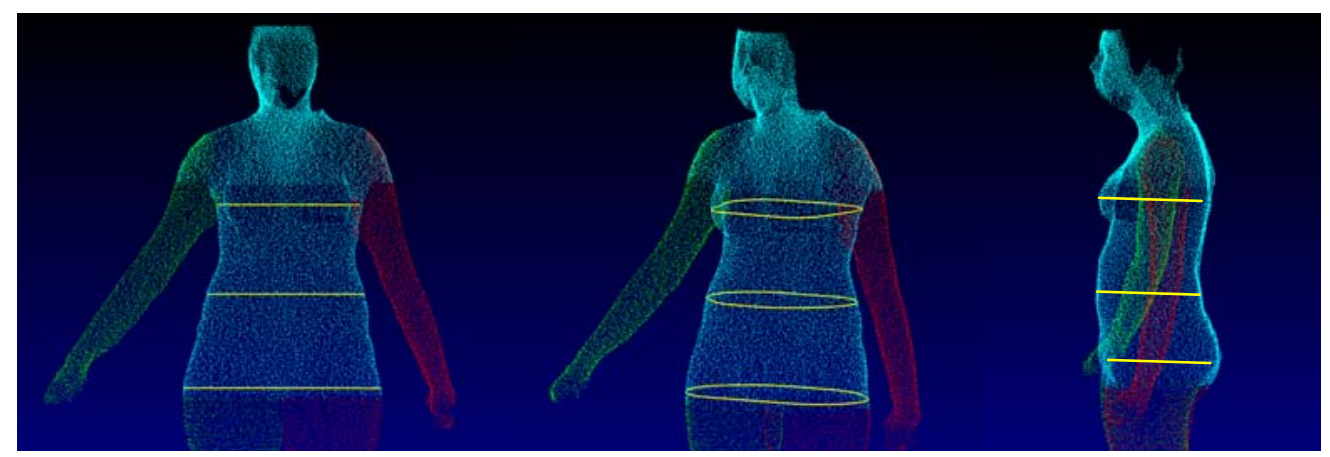

Fig. 3. Measurement extraction positions for the chest, waist and hips circumference, width and depth.

\subsection{Data analysis}

The data analysis was conducted in four stages,

1. Categorisation of each individual into body shapes based on the differences in width measurements at the chest, waist and hips

2. Categorisation of each individual into body shapes based on the differences in circumference measurements at the chest, waist and hips.

3. Comparison between the two methods of categorisation

4. Assessment of shape change with size increase.

Initially a set of rules for categorisation were developed and used (Table 1), segregating the population based on the difference between the chest, waist and hip width measurements. Each category was based on the differences between each of the three measurements, giving each category a unique code. For example, if the chest is at least $15 \%>$ than the waist (category 1 ), the hips are at least $15 \%>$ than the waist (category 1) and there is $<10 \%$ difference between the hips and chest (category 3 ) the individuals classification would be 1,1,3. Each classification was unique with regards to measurement ratios.

Table1. Classification of body shape rules.

\begin{tabular}{|c|c|c|c|c|c|c|}
\hline & & \multicolumn{5}{|c|}{ Category } \\
\hline $\begin{array}{c}\text { Body ratio } \\
\text { Number }\end{array}$ & Body ratio & 1 & 2 & 3 & 4 & 5 \\
\hline 1 & Chest to Waist & $\begin{array}{l}\text { Chest } 15 \% \\
>\text { Waist }\end{array}$ & $\begin{array}{l}\text { Chest } 10-15 \% \\
>\text { Waist }\end{array}$ & $<10 \%$ difference & $\begin{array}{l}\text { Waist } 10 \% \\
>\text { Chest }\end{array}$ & $\begin{array}{l}\text { Waist 10-15 } \\
>\text { Chest }\end{array}$ \\
\hline 2 & Waist to Hips & $\begin{array}{l}\text { Hips 15\% } \\
>\text { Waist }\end{array}$ & $\begin{array}{l}\text { Hips } 10-15 \% \\
>\text { Waist }\end{array}$ & $<10 \%$ difference & $\begin{array}{l}\text { Waist } 10 \% \\
>\text { Hips }\end{array}$ & $\begin{array}{l}\text { Waist } 10-15 \% \\
>\text { Hips }\end{array}$ \\
\hline 3 & Chest to hips & $\begin{array}{l}\text { Chest 15\% } \\
>\text { Hips }\end{array}$ & $\begin{array}{l}\text { Chest } 10-15 \% \\
>\text { Hips }\end{array}$ & $<10 \%$ difference & $\begin{array}{l}\text { Hips 10\% } \\
>\text { Chest }\end{array}$ & $\begin{array}{l}\text { Hips } 10-15 \% \\
>\text { Chest }\end{array}$ \\
\hline
\end{tabular}


Once all accepted scans had been categorised based on the width measurements the data was reprocessed and categorised based on the differences in circumferences, utilising the same categorisation method.

The results from both categorisation methods were then analysed individually with regards to the distribution of the population across all categories and the variation in overall body shape between individuals within the same category. The shape variation was determined by comparing width to depth ratio for all individuals within each shape category.

Once the 2 methods of categorisation had been analysed individually they were compared with regards to shape frequency and differences in shape variation within each category. Finally, the relationship between size and shape was analysed to determine how the form of the body changes as size increases. To determine if there is a significant change in body shape as size increases the distribution of each group was considered with regards to chest, hip and waist circumference.

\section{Results}

\subsection{Method 1}

The initial data analysis focused on the categorisation of subjects based on their chest, waist and hip width ratios (silhouette shape). The results identified that nine categories encompassed $97 \%$ of the scanned population, of which $96 \%$ of those could be categorised into 1 of 4 body shapes. Table 2 identifies the criteria for each of the four shapes. The results identified that the largest percentage of the population was classed as either a bottom heavy hourglass or spoon, with these 2 shapes covering $79 \%$ of the population (figure 4). A further $17 \%$ of the population was classed as either an hourglass or rectangular shape.

Table 2. Shape category definitions for method 1.

\begin{tabular}{|c|c|c|c|}
\hline & Chest to waist & Waist to hips & Chest to hips \\
\hline & & & $<10 \%$ difference \\
\hline Hourglass & Chest $15 \%+>$ Waist & Waist $15 \%+<$ Hips & Chest $10 \%<$ Hips \\
\hline Bottom heavy hourglass & Chest $10 \%-15 \%>$ Waist & Waist $15 \%+<$ Hips & $<10 \%$ difference \\
\hline Rectangle & $<10 \%$ difference & $<10 \%$ difference & Chest $15 \%+<$ Hips \\
\hline Spoon & Chest $10 \%-15 \%<$ waist & Waist $10 \%-15 \%<$ Hips & \multicolumn{2}{|c}{. }
\end{tabular}

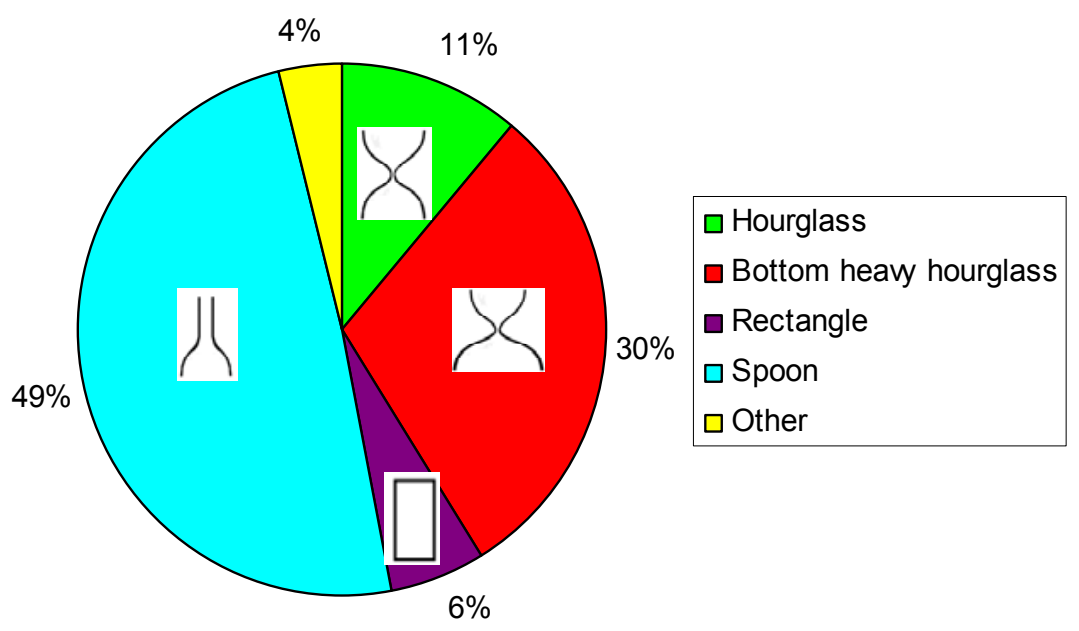

Fig 4. :Method 1 categorisation results.

These results were further analysed to determine if there were substantial shape differences within categories. A comparison of width to depth ratio at each measurement location was used to assess shape variation. As shown in Table 3 there is significant variation at each of the three measurement locations, despite being categorised as the same silhouette shape, with a maximum and mean variation of 1.66 and $0,51 \pm 0.37$ respectively across all measurements. 
Table 3. Maximum, minimum and mean width to depth ratio for each category, based on method 1, for the chest, waist and hips.

\begin{tabular}{|c|c|c|c|c|c|c|c|c|c|c|c|c|}
\hline & \multicolumn{12}{|c|}{ Ratio of width to depth } \\
\hline & \multicolumn{3}{|c|}{ Spoon } & \multicolumn{3}{|c|}{$\begin{array}{l}\text { Bottom heavy } \\
\text { hourglass }\end{array}$} & \multicolumn{3}{|c|}{ Hourglass } & \multicolumn{3}{|c|}{ Rectangle } \\
\hline & Min & Mean & Max & Min & Mean & Max & Min & Mean & Max & Min & Mean & Max \\
\hline Chest & 0.51 & 1.53 & 2.17 & 0.75 & 1.11 & 1.35 & 0.72 & 0.84 & 1.03 & 0.81 & 0.88 & 0.99 \\
\hline Waist & 0.83 & 1.02 & 1.23 & 0.81 & 1.18 & 1.53 & 0.69 & 0.79 & 1.11 & 1.01 & 1.12 & 1.16 \\
\hline Hips & 0.75 & 0.96 & 1.19 & 0.82 & 1.13 & 1.35 & 0.81 & 1.21 & 1.5 & 0.91 & 0.96 & 1.03 \\
\hline
\end{tabular}

\subsection{Method 2}

The results for the second method of categorisation were analysed in the same way as those from method 1. The results identified that 11 categories encompassed $95.7 \%$ of the population and that 4 shapes accounted for $95 \%$ of those (defined in Table 3 ). The shapes identified within this method were defined as Hourglass and soft hourglass, representing $70 \%$ of the population and rectangle and spoon incorporating a further $25 \%$. The analysis identified that the shape variation across each of the 4 primary shapes was relatively small, with the largest variation in width to depth ratio being 0.55 and an average variation of $0.31 \pm 0.14$ (Table 5).

Table 4. Shape category definitions for method 2.

\begin{tabular}{|c|c|c|c|}
\hline & Chest to waist & Waist to hips & Chest to hips \\
\hline & & & $<10 \%$ difference \\
\hline Hourglass & Chest $15 \%+>$ Waist & Waist $15 \%+<$ Hips & $<10 \%$ difference \\
\hline Soft hourglass & Chest $10 \%-15 \%>$ Waist & Waist $10 \%-15 \%<$ Hips & $<10 \%$ difference \\
\hline Rectangle & $<10 \%$ difference & $<10 \%$ difference & Chest $15 \%<$ Hips \\
\hline Spoon & Chest $10 \%<$ waist & Waist $10 \%<$ Hips & Chis
\end{tabular}

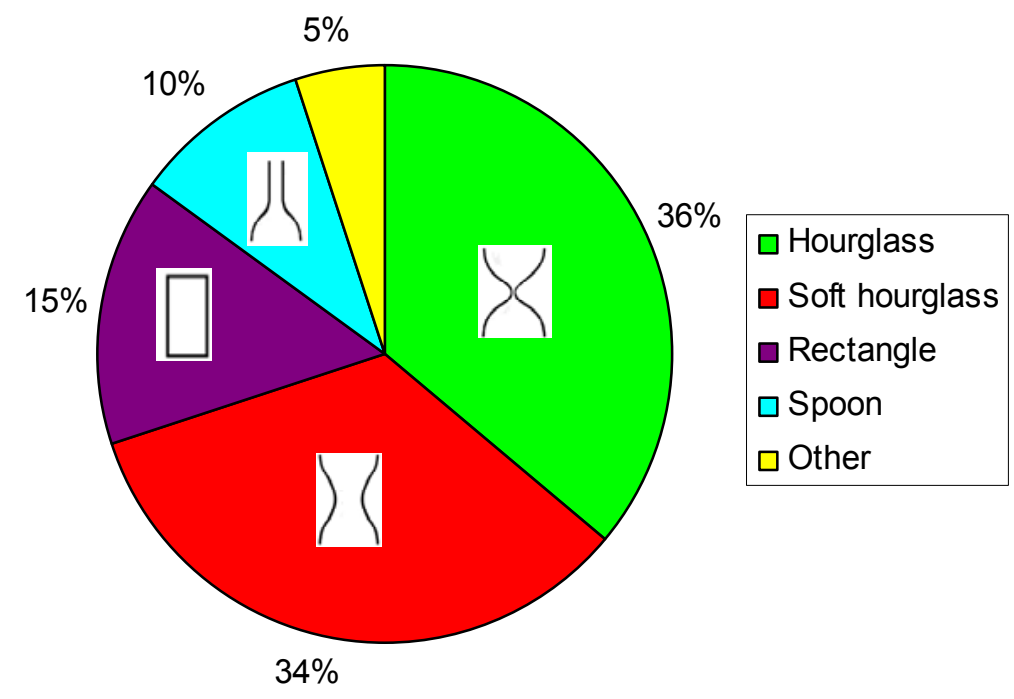

Fig. 5. Method 2 categorisation results.

Table 5. Maximum, minimum and mean width to depth ratio for each category, based on method 2, for the chest, waist and hips.

\begin{tabular}{|c|c|c|c|c|c|c|c|c|c|c|c|c|}
\hline & \multicolumn{12}{|c|}{ Ratio of width to depth } \\
\hline & \multicolumn{3}{|c|}{ Spoon } & \multicolumn{3}{|c|}{$\begin{array}{l}\text { Bottom heavy } \\
\text { hourglass }\end{array}$} & \multicolumn{3}{|c|}{ Hourglass } & \multicolumn{3}{|c|}{ Rectangle } \\
\hline & Min & Mean & Max & Min & Mean & Max & Min & Mean & Max & Min & Mean & Max \\
\hline Chest & 0.87 & 1.14 & 1.42 & 0.86 & 1.09 & 1.21 & 0.81 & 0.89 & 0.99 & 0.92 & 0.99 & 1.11 \\
\hline Waist & 0.95 & 1.06 & 1.17 & 0.82 & 1.12 & 1.33 & 0.92 & 1.10 & 1.13 & 0.98 & 1.08 & 1.12 \\
\hline Hips & 0.88 & 1.02 & 1.20 & 0.88 & 1.11 & 1.22 & 0.91 & 1.25 & 1.42 & 0.97 & 1.05 & 1.07 \\
\hline
\end{tabular}




\subsection{Comparison of methods}

The results from method 1 and 2 show significant differences with regards to the grouping of individuals and the variation in body shapes within the same category. A key difference between the results for each of the two methods is the identification of different primary body shapes. Method 1 identified a substantial percentage of the population as bottom heavy hourglasses $(30 \%)$, and less than $1 \%$ as soft hourglasses, whereas, method 2 identified $34 \%$ as soft hourglasses and $2.3 \%$ as bottom heavy hourglasses.

Another significant difference between the two methods was with regards to the spoon and hourglass categories, with method 1 identifying $49 \%$ and $10 \%$ of the population as spoon or hourglass shape respectively, compared to $10 \%$ and $36 \%$ when using method 2 .

With regards to the shape consistency within categories, the comparison between width and depth measurements identified that categorisation through the use of method 1 resulted in substantially more variation in width to depth ratio, 0.55 compared to 0.31 for method 2, and demonstrates increased variation in 16 out of 18 cases (Table 6). These results suggest that the overall shape of the individual has greater variation when categorised according to the silhouette shape, which could have detrimental affects on clothing fit if designed based on these categorisations.

Table 6. Comparison of the range of width to depth ratio for each shape category, based on method 1 for the chest, waist and hips

\begin{tabular}{|c|c|c|c|c|c|c|c|c|c|c|}
\hline & \multicolumn{10}{|c|}{ Depth to width ratio range } \\
\hline & \multicolumn{2}{|c|}{ Spoon } & \multicolumn{2}{|c|}{$\begin{array}{c}\text { Bottom heavy } \\
\text { hourglass }\end{array}$} & \multicolumn{2}{|c|}{ Soft hourglass } & \multicolumn{2}{|c|}{ Hourglass } & \multicolumn{2}{|c|}{ Rectangle } \\
\hline & $\begin{array}{c}\text { Method } \\
1 \\
\end{array}$ & $\begin{array}{c}\text { Method } \\
2 \\
\end{array}$ & $\begin{array}{c}\text { Method } \\
1 \\
\end{array}$ & $\begin{array}{c}\text { Method } \\
2 \\
\end{array}$ & $\begin{array}{c}\text { Method } \\
1 \\
\end{array}$ & $\begin{array}{c}\text { Method } \\
2 \\
\end{array}$ & $\begin{array}{c}\text { Method } \\
1 \\
\end{array}$ & $\begin{array}{c}\text { Method } \\
2 \\
\end{array}$ & $\begin{array}{c}\text { Method } \\
1\end{array}$ & $\begin{array}{c}\text { Method } \\
2 \\
\end{array}$ \\
\hline Chest & 1.66 & 0.55 & 0.60 & 0.31 & 0.74 & 0.35 & 0.31 & 0.18 & 0.18 & 0.19 \\
\hline Waist & 0.4 & 0.22 & 0.72 & 0.28 & 0.82 & 0.51 & 0.42 & 0.21 & 0.15 & 0.14 \\
\hline Hips & 0.44 & 0.38 & 0.53 & 0.31 & 0.44 & 0.34 & 0.69 & 0.51 & 0.12 & 0.1 \\
\hline Mean & 0.83 & 0.38 & 0.62 & 0.30 & 0.67 & 0.4 & 0.47 & 0.3 & 0.15 & 0.14 \\
\hline $\begin{array}{l}\text { standard } \\
\text { deviation }\end{array}$ & 0.72 & 0.17 & 0.10 & 0.02 & 0.20 & 0.10 & 0.20 & 0.18 & 0.03 & 0.05 \\
\hline
\end{tabular}

\subsection{Development of sizing system}

The results discussed in the previous sections identified that categorisations based on silhouette shape can result in greater variation in form between individuals identified as having the same overall body shape. Therefore, this next section will utilise the results of method 2 to analyse shape variation across sizes, as this has been found to be the more repeatable method of categorisation.

To determine if there was a relationship between size and shape all the results for each category were compared, with regards to each of the three measurements. The results highlight that as circumference increased the shape classification changed (figure 6). This was particularly evident for the waist measurement.

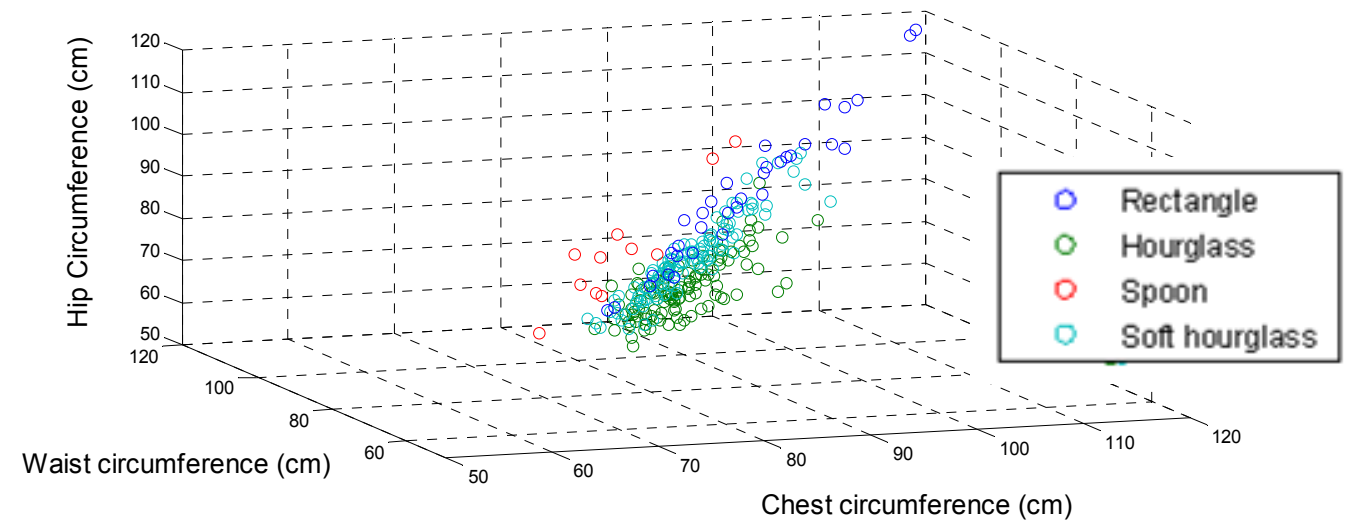

Fig. 6. Chest, waist and hip measurements for all subjects categorised within their respective shape. 
To further determine the relationship between size and shape the distribution of the population within each category was analysed with regards to increases in circumference for each of the three measurements. The results indicate that there is a gradual change in body shape as size increases (Figure 7). The results suggest that as size increases the chest to waist and waist to hip ratio decreases. As a result the most representative shape of the population gradually changes from an hourglass to a rectangular shape as size increases.
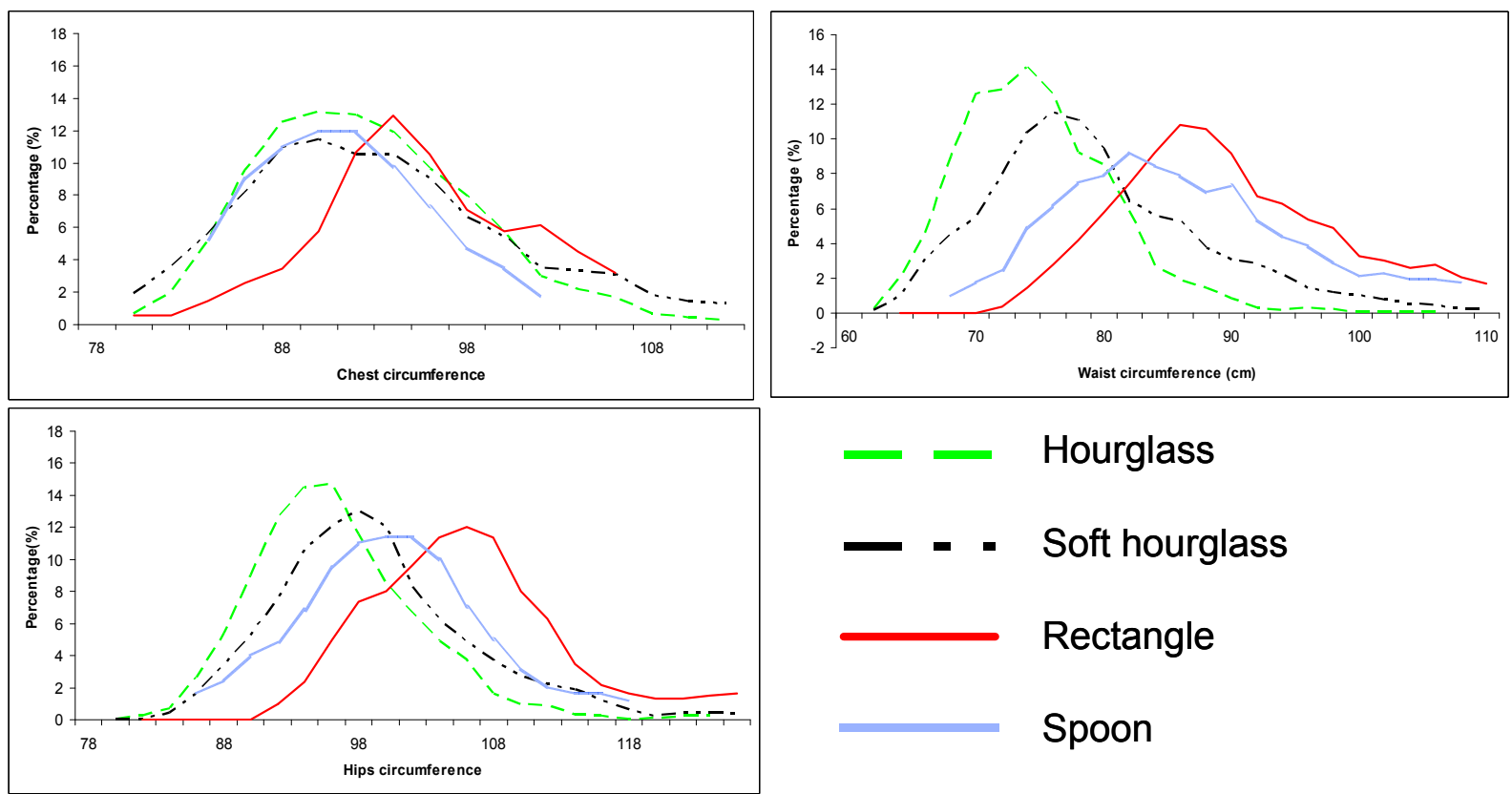

Hourglass

Rectangle

Spoon

Fig. 7. Distribution of the population within each shape category based on method 2.

\section{Discussion}

Body shape identification is a key factor in developing appropriate clothing sizing systems for women. Currently studies focus on the assessment of silhouette shape when categorising the female form [7]. This paper aimed to assess the appropriateness of this method and compare it to the use of circumference measurements. The results identified significant differences between both methods when analysing the same population. These differences included identification of different shapes based on the ratio between chest, waist and hip measurements. The silhouette based method identified four primary categories, hourglass, bottom heavy hourglass, spoon and rectangle, whereas, the circumference based method identified hourglass, soft hourglass, spoon and rectangle as the four predominant shapes. As well as identifying different categories, the percentage of the population within each shape varied. To assess the appropriateness of both methods, the variation within each category was assessed with regards to the overall shape at each measurement location. The results identified that there was substantially more variation when categorised using method 1 . From this it was determined that method 2 was most appropriate for the development of sizing systems.

The final part of this paper then used the results from method two to identify how shape and size are related. The results highlighted that as size increases the shape of the individual generally changes from hourglass to rectangular, with the chest to waist and hip to waist ratio decreasing as size increases.

In conclusion, this paper has demonstrated that through the use of circumference rather than width measurements the female population can be categorised into different body shape groups, with reduced variation within each category. Finally, it is apparent that within clothing sizing systems non-linear gradation of the chest, waist and hips must be used, with a particular focus on gradually reducing chest to waist and waist to hip difference to increase fit within the larger sizes where the population is more of a rectangular shape rather than hourglass as found in the smaller sizes. 


\section{References}

1. Fan, J., Yu, W. and Hunter, L. (2004), "Clothing Appearance and Fit: Science and Technology", Woodhead, Cambridge.

2. Waugh, C.F. (1999). "Well-cut through the body: Fitted clothing in twelfth-century Europe", Dress, 26, 3-17.

3. Loker, S., Ashdown, S. and Schoenfelder, K. (2005), "Size-specific analysis of body scan data to improve apparel fit", Journal of Textile and Apparel Technology and Management, Vol. 4 No. 3, pp. 1-15.

4. Keiser, S.J., and Gaener, M.B., (2003) "Beyond design, the synergy of apparel product development" In Sizing and fit, pp 301- 324.

5. DesMarteau, K. (2000) "CAD: Let the fit revolution begin. Bobbin, 42, 42-56.

6. Vuruskan, A. and Bulgun, E., (2011): "Identification of female body shapes based on numerical evaluations", International Journal of Clothing Science and Technology, Vol. 23 No. 1 